\title{
Atomic pair distribution function analysis of materials containing crystalline and amorphous phases
}

\author{
Thomas Proffen*, I, Katharine L. Page ${ }^{\mathrm{I}}$, Sylvia E. McLain ${ }^{\mathrm{I}}$, Bjørn Clausen ${ }^{\mathrm{I}}$, Timothy W. Darling ${ }^{\mathrm{II}}$, James A. TenCate ${ }^{\mathrm{III}}$, \\ Seung-Yub Lee ${ }^{\mathrm{IV}}$ and Ersan UstundagV \\ I Lujan Neutron Scattering Center, Los Alamos National Laboratory, MS H805, Los Alamos, NM 87545, USA \\ II Material Science and Technology, Los Alamos National Laboratory, MS K764, Los Alamos, NM 87545, USA \\ III Earth and Environmental Science, Los Alamos National Laboratory, MS D443, Los Alamos, NM 87545, USA \\ ${ }^{\text {IV }}$ Department of Materials Science, California Institute of Technology, Pasadena, CA 91125, USA \\ v Department of Materials Science and Engineering, Iowa State University, Ames, IA 50011, USA
}

Received November 23, 2004; accepted April 6, 2005

Pair Distribution Function / Disorder / Neutron scattering / Powder diffraction / Bulk metallic glass / Quartz

\begin{abstract}
The atomic pair distribution function (PDF) approach has been used to study the local structure of liquids, glasses and disordered crystalline materials. In this paper, we demonstrate the use of the PDF method to investigate systems containing a crystalline and an amorphous structural phase. We present two examples: Bulk metallic glass with crystalline reinforcements and Fontainebleau sandstone, where an unexpected glassy phase was discovered. In this paper we also discuss the refinement methods used in detail.
\end{abstract}

\section{Introduction}

The understanding and possible prediction of interesting macroscopic properties of modern materials relies heavily on the precise knowledge of their atomic structure. For more than 100 years, crystallographic methods have allowed us to extract structural information from Bragg reflections. Remarkable progress in crystallography allows for the structures of complex biological molecules, such as proteins, to be routinely solved. At the same time the growing complexity of materials has shown limits of the crystallographic approach since it only yields the average structure of the material as it is based on the analysis of Bragg intensities. However, deviations from the average structure result in diffuse scattering which in general contains two-body atomic correlations and thus holds the key to the defect or local structure of the material. Diffuse scattering is in fact the topic of this special issue of Zeitschrift für Kristallographie. One approach to unravel the defect structure is the study of single crystal diffuse scattering which is covered in some of the other papers in this special issue and also in detail in two recent books by Nield and Keen (2001) and

\footnotetext{
* Correspondence author (e-mail: tproffen@lanl.gov)
}

Welberry (2004). More information as well as all the software used in the analysis presented in this paper can be found at Billinge and Proffen (2004).

We use the pair distribution function (PDF) approach to study the local structure of materials. The PDF is obtained from a total scattering powder diffraction pattern via a Fourier transform. Since the total scattering pattern is composed of Bragg as well as diffuse scattering contributions, it contains local, medium range and long range structural information. Details about the method and its application to disordered materials can be found in a recent review (Proffen et al., 2003). In previous papers, we have investigated the application of the PDF method to study chemical short-range order (Proffen, 2000; Proffen et al., 2002b) and the study of domain structures using PDF (Proffen und Page, 2004). In this paper we continue this series of systematic investigations of the PDF method and ask the question, how can the PDF method be applied to systems where crystalline and amorphous structural phases co-exist?

\section{The PDF method}

Before discussing the specific application to materials containing amorphous and crystalline phases, we give a short summary of the PDF method from carrying out the experiment to data modeling. Many more details about the PDF method as well as its application can be found in the book by Egami and Billinge (2003).

As mentioned above, the PDF, $G(r)$, is obtained via the Fourier Transform of the total diffraction pattern as indicated below,

$$
\begin{aligned}
G(r) & =4 \pi r\left[\rho(r)-\rho_{0}\right] \\
& =\frac{2}{\pi} \int_{0}^{\infty} Q[S(Q)-1] \sin (Q r) \mathrm{d} Q,
\end{aligned}
$$

where $\rho(r)$ is the microscopic pair density, $\rho_{0}$ is the average number density and $Q$ is the momentum transfer 
$(Q=4 \pi \sin (\Theta) / \lambda) . \quad S(Q)$ is the normalized structure function determined from the experimental diffraction intensity (Egami and Billinge, 2003). All data presented in this paper were collected on the recently upgraded high resolution neutron powder diffractometer NPDF (Proffen et al., 2002a) at the Lujan Neutron Scattering Center at Los Alamos National Laboratory. The data are corrected for detector deadtime and efficiency, background, absorption, multiple scattering, inelasticity effects and normalized by the incident flux and the total sample scattering crosssection to yield the total scattering structure function, $S(Q)$ and subsequently $G(r)$. Data processing was carried out using the program PDFgetN (Peterson et al., 2000).

Having obtained the experimental PDF, one usually wants to refine a structural model. Two approaches can be taken: In cases where the disordered model requires a large number of atoms, typically tens of thousands, the Reverse Monte Carlo technique (McGreevy and Pusztai, 1988) can be used to match a structural model containing many atoms to an experimental PDF. A comprehensive summary of the method applied to crystalline materials is given in Tucker et al. (2001). In cases where a smaller local structural picture is sufficient, least-squares full profile refinement of the PDF can be used to obtain a structural description. This is the approach we use in this paper. Considering the PDF is related to the bond length distribution of the material weighted by the respective scattering powers of the contributing atoms, it can easily be calculated using the relation

$$
G(r)=\frac{1}{r} \sum_{i} \sum_{j}\left[\frac{b_{i} b_{j}}{\langle b\rangle^{2}} \delta\left(r-r_{i j}\right)\right]-4 \pi r \rho_{0},
$$

where the sum goes over all pairs of atoms $i$ and $j$ within the model crystal separated by $r_{i j}$. The scattering power of atom $i$ is $b_{i}$ and $\langle b\rangle$ is the average scattering power of the sample. In a multi phase system, the total PDF is

$$
G(r)=\sum_{p} G_{p}(r)
$$

where $G_{p}(r)$ is the PDF of the structural phase $p$ and the sum goes over all phases $p$ present in the model. It should be noted, that this assumes that the size of each phase is large compared to the range $r$ refined. To account for the cutoff of $S(Q)$ at $Q_{\max }$, the calculated function $G(r)$ is then convoluted with a termination function, $\sin \left(Q_{\max } r\right) / r$. Refinements presented in this paper were carried out using the program PDFFIT (Proffen and Billinge, 1999). The program allows structural parameters such as lattice parameters, anisotropic atomic displacement parameters, position and site occupancies to be refined. Even though this is similar to the results of a Rietveld refinement, one needs to realize that the structural model obtained is strictly only valid for length scales corresponding to the $r$-range used for the refinement. This opens up the possibility to study the local structure on different length scales by varying the $r$ range refined. In addition to structural parameters, there are two other corrections to the calculated PDF: First the finite resolution, $\Delta Q$ of the instrument leads to a dampening of the PDF intensities by $\exp \left(-\Delta Q^{2} r^{2} / 2\right)$. The second correction accounts for changes in the PDF peak width.

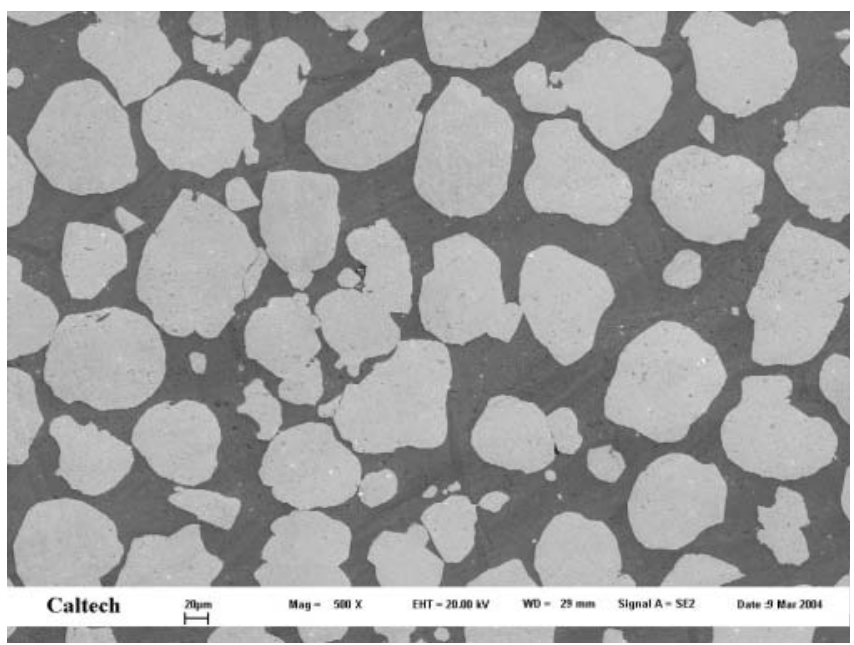

Fig. 1. Image of a cross section of the composite sample at a magnification of 500 times. The dark regions are the BMG matrix and the light regions are the tungsten reinforcements.

The PDF peak width for a pair of atoms $i$ and $j$ is calculated as (Proffen and Billinge, 2003)

$$
\sigma_{i j}=\sqrt{\sigma_{i j}^{\prime 2}-\frac{\delta}{r_{i j}^{2}}-\frac{\gamma}{r_{i j}}+\alpha^{2} r_{i j}^{2}}
$$

The first term $\sigma_{i j}^{\prime}$ is the PDF peak width of the structural component due to the atomic displacement parameters. The next two parameters, $\delta$ and $\gamma$, determine the sharpening of near neighbor PDF peaks due to correlated motion, in other words the tendency to move in phase (see Jeong et al. (2003, 1999)). Finally the parameter $\alpha$ determines the PDF peak broadening at very high distances $r$ due to the instrument resolution.

\section{Example I: Bulk metallic glass composite}

Recently developed bulk metallic glass (BMG) alloys (Peker and Johnson, 1993) have attractive mechanical properties for structural applications: yield strength around 2 $\mathrm{GPa}$, fracture toughness above $20 \mathrm{MPa} \sqrt{m}$, good corrosion resistance and specific strength (Gilbert et al., 1993; Bruck et al., 1994; Waniuk et al., 2001). Unfortunately, BMGs fail catastrophically by formation of macroscopic shear bands during unconstrained deformation at room temperature (Gilbert et al., 1993; Bruck et al., 1994). To avoid this failure mode, BMG matrix composites have been developed where the reinforcements appear to inhibit the formation of a single, catastrophic shear band (Conner et al., 1998; Choi-Yim et al., 1999; Szuecs et al., 2001; Hays et al., 2001). However, the BMG matrix composites are then subject to development of residual and internal stresses due to the differences in material parameters between its constituents, as is the case for all composites. Several studies have sought to elucidate the residual stresses and the development of internal stresses during loading using a combination of diffraction techniques and modeling (Dragoi et al., 2001; Balch et al., 2003; Clausen et al., 2003). The amorphous structure of the BMG does 
not lend itself to classical elastic powder scattering as it does not give rise to Bragg diffraction peaks. Therefore the previous determination of internal stresses in the BMG matrix composites have been based upon measurements in the crystalline second phase and model predictions for the BMG. In the present work we have employed the PDF method to obtain information from both the crystalline second phase, in this case tungsten particles, and the BMG matrix. The Vit106 ${ }^{\complement}$ BMG alloy was used as a matrix material for composites. Ingots of the alloy were prepared by arc melting a mixture of the elements having a purity of 99.7 at $\%$ or better. In order to fabricate particulate composite with high volume fraction, particles were placed in the sealed end of a $10 \mathrm{~mm}$ I.D. 304 stainless steel tube, which was necked at $2 \mathrm{~cm}$ above the reinforcement, and ingots of the matrix material were put into the tube above the neck. The volume fraction of tungsten particles was $60 \% \pm 2.5 \%$, and average particle size was about $80 \mu \mathrm{m}$ (Fig. 1). The sample preparation procedures are described in detail in the paper by Choi-Yim et al. (2002).

Two samples were measured on NPDF: Pure Vit106 ${ }^{(\mathcal{C}}$ bulk metallic glass and a composite of Vit106 ${ }^{\odot}$ and $60 \%$ tungsten particles. The samples were measured for 11 and 16 hours respectively. The resulting reduced structure functions $F(Q)=Q[S(Q)-1]$ are shown in Fig. 2. The upper curve shows the data for the composite sample and it is clearly dominated by the crystalline diffraction pattern of tungsten. The lower curve shows the data for the pure glass, obviously showing no Bragg peaks. The data were

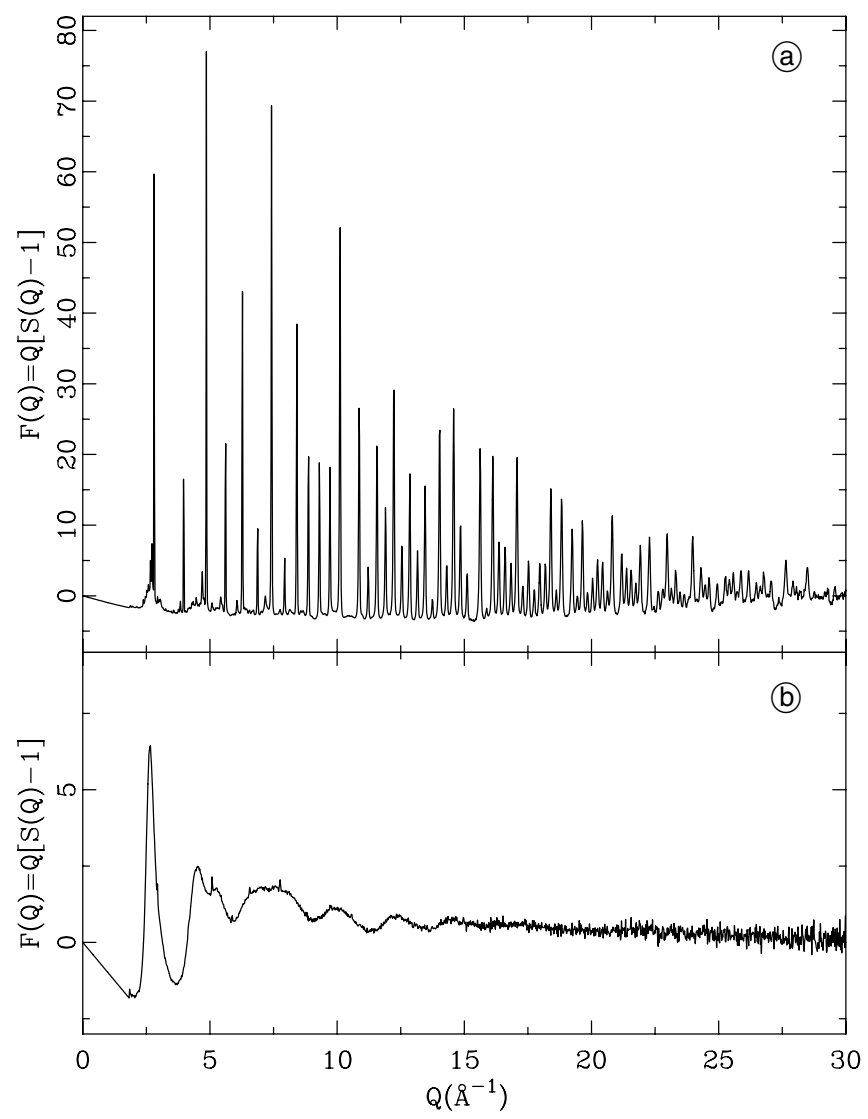

Fig. 2. Reduced structure function $F(Q)=Q[S(Q)-1]$ of (a) the Vit106 ${ }^{\mathbb{C}}-\mathrm{W}$ composite sample and $(\mathbf{b})$ the Vit106 ${ }^{\complement}$ glass sample.

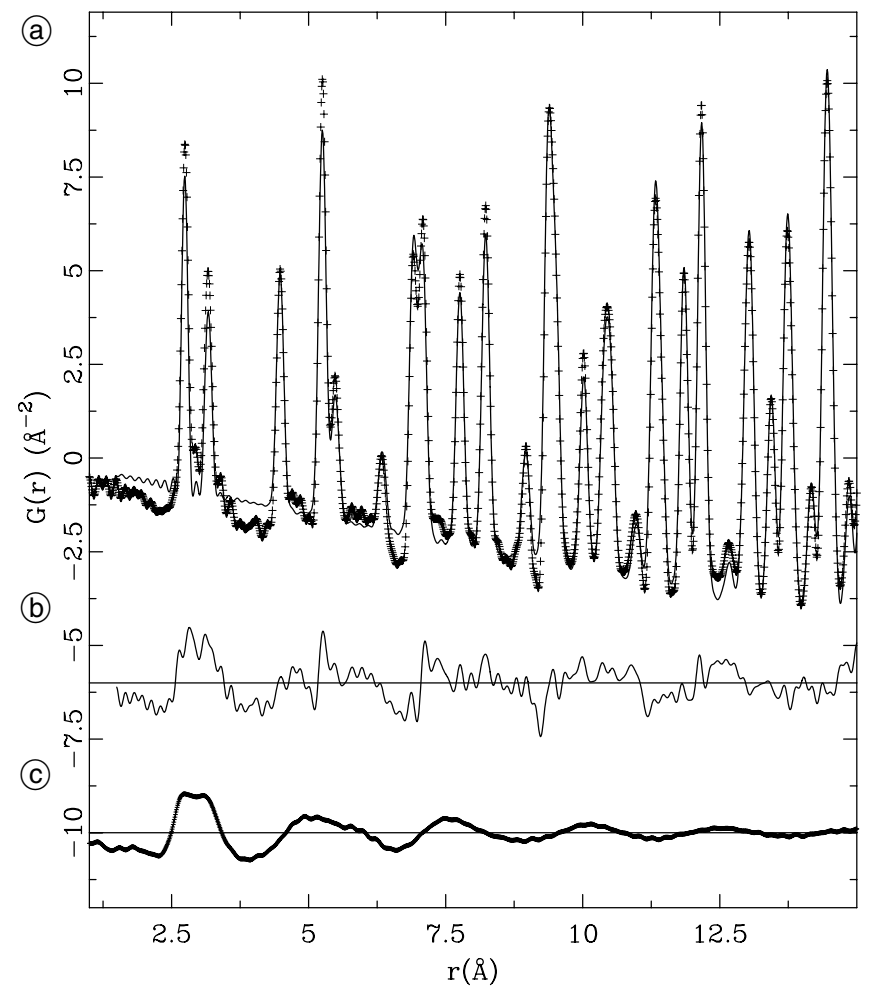

Fig. 3. (a) PDF refinement result for the Vit $106^{\mathrm{C}}-\mathrm{W}$ composite sample. The crosses mark the experimental PDF and the solid line the calculated PDF for the crystalline W phase, (b) shows the difference between model and data and (c) shows the scaled experimental PDF of the Vit106 ${ }^{\complement}$ sample.

terminated at $Q=35 \AA^{-1}$ to obtain the corresponding PDFs, $G(r)$ (Fig. 3a). Next a model of crystalline tungsten was refined and the result is shown in Fig. 3a. The space group is $\operatorname{Im} 3 m$, the refined lattice parameter is $a=3.1684(1) \AA$ and the atomic displacement parameter is $\left\langle u^{2}\right\rangle(\mathrm{W})=0.002574 \AA^{2}$. Closer inspection of Fig. 3a reveals significant differences between the calculated and observed PDF at low distances $r$. This is evident in the difference curve shown as solid line below the data and refinement in Fig. 3b. This difference curve follows the experimental PDF of the pure Vit106 ${ }^{\odot}$ glass shown in Fig. 3c. These data were scaled by the volume fraction of $40 \%$ and its agreement with the difference curve or the PDF intensities not accounted for by the crystalline tungsten is quite remarkable. At this point we see the amorphous component of the composite material, but the next step is to model the amorphous component or even the composite as a whole. This is part of our ongoing efforts.

\section{Example II: Evidence for vitreous silica in sandstone}

The previous example illustrated that we can in fact separate the crystalline and amorphous components in the PDF. In this example we present a quantitative analysis of PDF data of Fontainebleau sandstone, where an unexpected amorphous phase was discovered. Here we focus on the modeling aspects, for more details see Page et al. (2004).

Crystallographically the structure of many consolidated sandstones appears to be mainly quartz. However, peculiar 


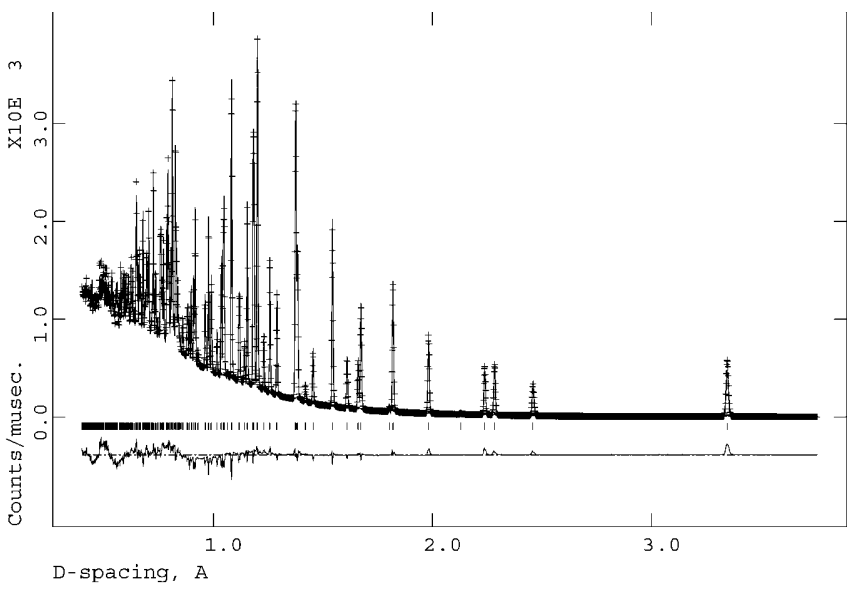

Fig. 4. Rietveld refinement result for Fontainebleau sandstone. Shown are the data for the 90 degree bank of NPDF.

nonlinear properties have been observed in sandstone (TenCate and Shenkland, 1996; Darling et al., 2004), that are not found in pure quartz. We carried out PDF measurements in an effort to find a local structural explanation for these properties. Measurements were carried out on a cylindrical piece of Fontainebleau sandstone, approximately $50 \mathrm{~mm}$ high with a diameter of $9 \mathrm{~mm}$, sealed in a vanadium can. The measuring time was 24 hours which resulted in very high quality data. As a first step we preformed Rietveld refinements using the GSAS package (Larson and von Dreele, 2000). The result is shown in Fig. 4 and the refined structural parameters are listed in Table 1. The resulting structure is in good agreement with literature values. Inspection of Fig. 4 also reveals that there are no additional Bragg peaks present, in other words the average structure of the Fontainebleau sandstone is pure crystalline quartz. One might note the modulation of the background visible at low values of $d$ in Fig. 4.

Next the data were processed to obtain a reduced structure function $F(Q)=Q[S(Q)-1] \quad$ (Fig. 5). Note that $F(Q)$ above $Q=20 \AA^{-1}$ is enlarged by a factor of three. The high quality or low noise level of the data is easily visible. In addition a significant modulation of the scattering data at high $Q$ can be observed. The data were terminated at $Q_{\max }=40 \AA^{-1}$ to obtain the corresponding PDF shown as crosses in Fig. 6. The solid line in the top panel of Fig. 6 corresponds to a refined crystalline quartz model over the range of $1<r<20 \AA$. These refinements started from the structural result of the Rietveld refinement. Then a scale factor, lattice parameters and atomic displacement parameters were refined using PDFFIT. The final values are listed in the left column of Table 2. Two observations

Table 1. Results of Rietveld refinement. The space group is $P 3_{1} 21$ and the resulting lattice parameters are $a=4.91444(1) \AA$ and $c=5.40646(3) \AA$. The units of $\left\langle u^{2}\right\rangle$ are given in $\AA^{2}$ and multiplied by 1000 . The numbers in parentheses are the estimated standard deviation on the last digit.

\begin{tabular}{lllll}
\hline Atom & $x$ & $y$ & $z$ & $\left\langle u^{2}\right\rangle$ \\
\hline $\mathrm{Si}$ & $0.46954(11)$ & 0.0 & $1 / 3$ & $7.86(8)$ \\
$\mathrm{O}$ & $0.41300(9)$ & $0.26744(8)$ & $0.21437(6)$ & $13.30(5)$ \\
\hline
\end{tabular}

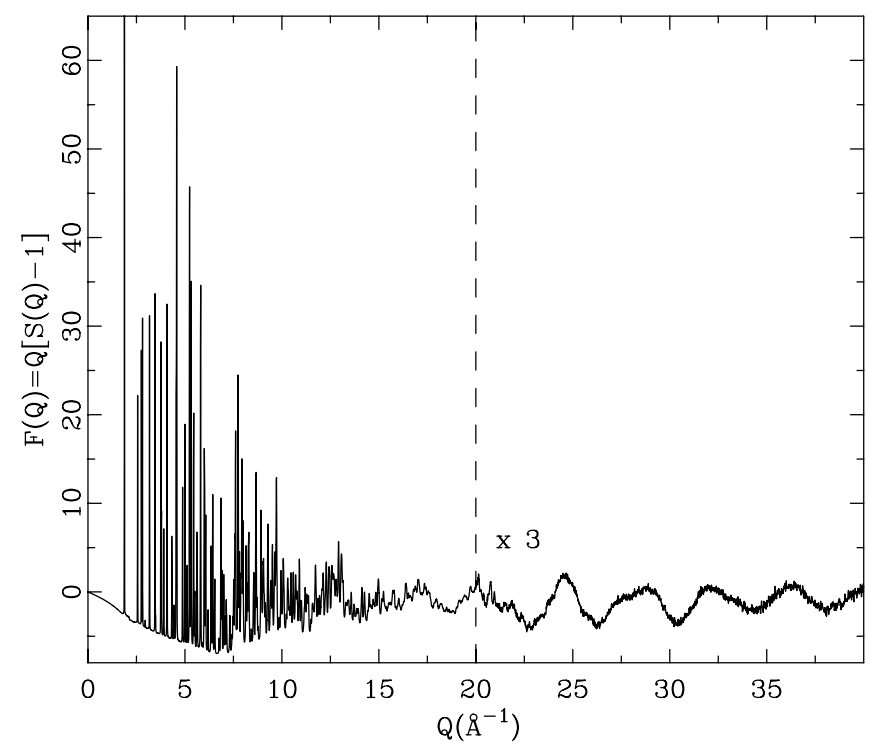

Fig. 5. Reduced structure function $F(Q)=Q[S(Q)-1]$ for the Fontainebleau sandstone measurement on NPDF. Data above $Q=20 \AA^{-1}$ are enlarged by a factor of three. The strongest peak is cut off for clarity. It extends to $F(Q)=137.8$.

can be made from inspecting the top panel of Fig. 6: First the agreement between model and experimental PDF at distances with $r>3 \AA$ is excellent and secondly the agreement for $r<3 \AA$ is very poor. This is also reflected

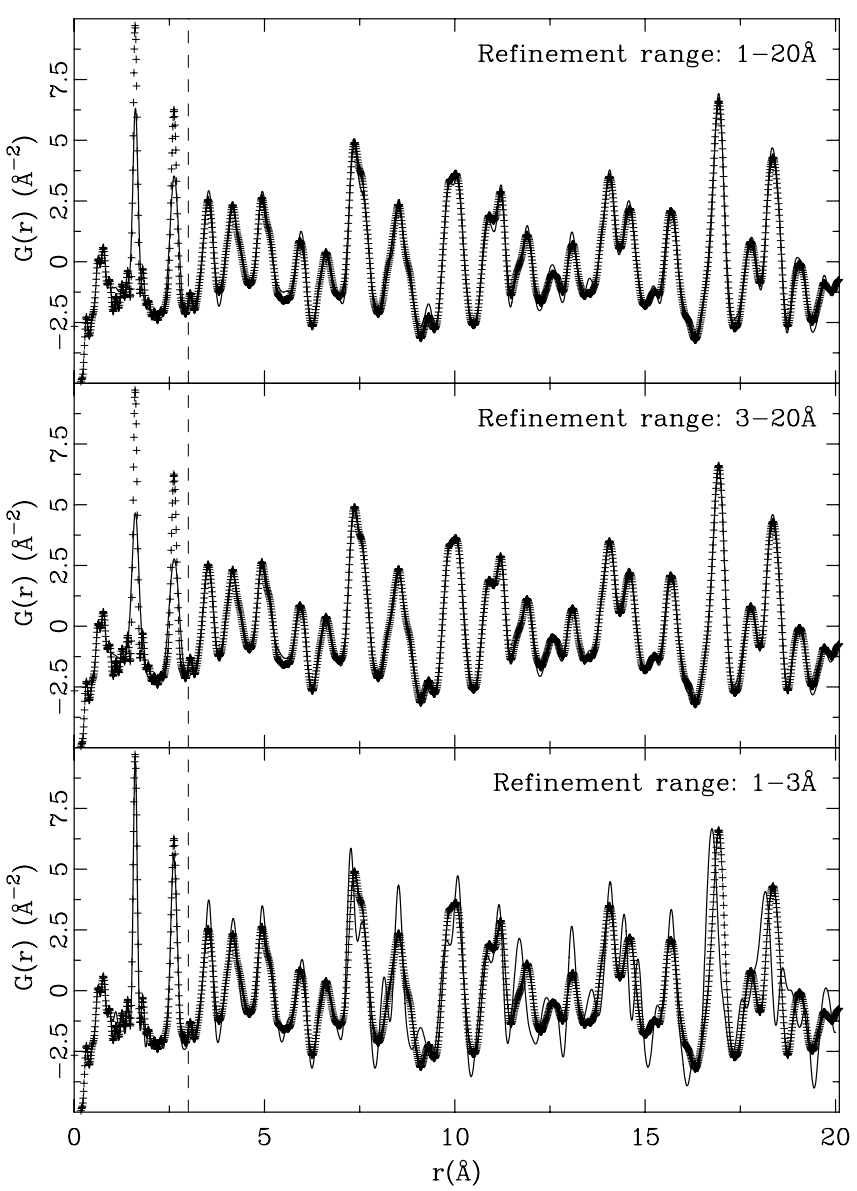

Fig. 6. PDF refinement results using a single quartz phase. The refinement range is indicated in each panel. After refining, the PDF for the complete range $1<r<20 \AA$ was calculated from the resulting structural model. The vertical dashed line indicates $r=3 \AA$. 
Table 2. PDF results of single phase refinements for different refinement ranges $r$. The units for the lattice parameters are $\AA$ and the values of $\left\langle u^{2}\right\rangle$ are given in $\AA^{2}$ and multiplied by 1000. The numbers in parentheses are the estimated standard deviation on the last digit. The correlated motion parameter was fixed to $\delta=0.018$. Atomic coordinates are taken from the Rietveld refinement (see text).

\begin{tabular}{llll}
\hline Refinement range & $1<r<20 \AA$ & $3<r<20 \AA$ & $1<r<3 \AA$ \\
\hline$a$ & $4.9198(1)$ & $4.9176(1)$ & $4.9320(7)$ \\
$c$ & $5.4044(2)$ & $5.4069(1)$ & $5.315(2)$ \\
$\left\langle u^{2}\right\rangle(\mathrm{Si})$ & $3.666(3)$ & $4.800(4)$ & $3.908(2)$ \\
$\left\langle u^{2}\right\rangle(\mathrm{O})$ & $7.500(3)$ & $10.303(4)$ & $4.777(1)$ \\
$R_{w p}(1-20 \AA)$ & $20.6 \%$ & $23.7 \%$ & $64.8 \%$ \\
$R_{\mathrm{wp}}(3-20 \AA)$ & $14.5 \%$ & $9.6 \%$ & $72.5 \%$ \\
$R_{w p}(1-3 \AA)$ & $35.1 \%$ & $48.2 \%$ & $13.3 \%$ \\
Scale & $1.009(1)$ & $1.118(1)$ & $0.999(3)$ \\
\hline
\end{tabular}

in the weighted $R$-value, $R_{w p}$, listed in Table 2 for the different ranges. To further investigate this behavior, we carried out identical refinements for two additional refinement ranges of $3<r<20 \AA$ and $1<r<3 \AA$ shown in the center and bottom panel of Fig. 6. In this case, the agreement between model and data is good over the refined range, but poor outside it. Structural parameters as well as $R$-values for all three $r$-ranges are listed in Table 2. Obviously, the crystalline quartz model cannot describe the observed PDF of our sandstone over the complete distance range. However, the differences manifest themselves in an excess intensity of the first two PDF peaks, corresponding to the nearest $\mathrm{Si}-\mathrm{O}$ and $\mathrm{O}-\mathrm{O}$ distances. This is also confirmed when studying the scale factors for the different refinement ranges. If just the region containing the first two peaks is refined, the scale factor is one (Table 2) as expected for properly normalized data. However, for the range $3<r<20 \AA$ the scale factor needed to match the observed data is 1.1, and a good agreement between observed and calculated data is achieved over both regions. This in fact rules out local distortions or thermal diffuse scattering (TDS) as explanation since this type of disorder influences the shape and position of PDF peaks, but the total number of contributing atom pairs is conserved with respect to the perfect crystal. In other words no change in scale factor would be seen. The effect of TDS manifests itself in significantly smaller atomic displacement parameters, $\left\langle u^{2}\right\rangle$, when refining only over the range $1<r<3 \AA$, accounting for correlated motion as discussed earlier. Chemical short range order on the other hand changes the intensities of certain PDF peaks Proffen 2000, but differently on the first and second PDF peak. This again is not what we observe. A possible explanation is offered, by assuming that approximately $10 \%$ of the $\mathrm{SiO}_{4}$ tetrahedra do not participate in the crystalline long range ordered phase and consequently are not present in the observed PDF peaks above $r>3 \AA$. This is also consistent with the modulation observed at high $Q$. The nearest $\mathrm{Si}-\mathrm{O}$ distance of about $1.6 \AA$ results in the modulation in $Q$ of $2 \pi / 1.6=3.9 \AA^{-1}$ that can be observed in $F(Q)=Q(S(Q)-1)$ in Fig. 5 .

The next step is the refinement of a two phase structural model to account for the extra PDF intensities at low $r$

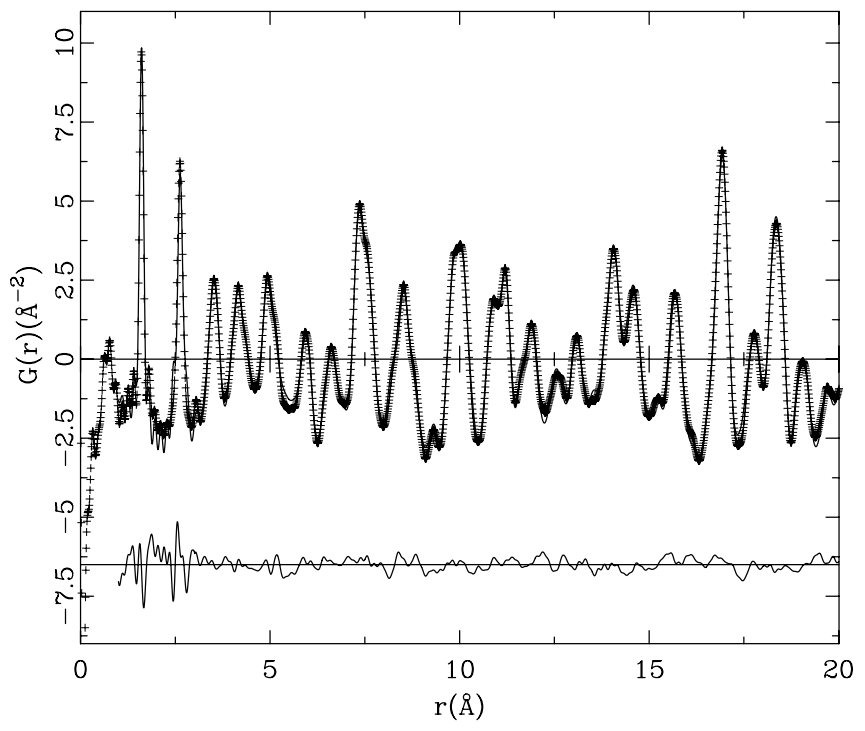

Fig. 7. PDF refinement results using a two phase model. The refinement range is $1<r<20 \AA$. See text for details.

values. The simplest approach is to construct each phase from the crystalline structure of quartz. However, to account for the missing long range order, one of the phases creates correlations only out to a cutoff value, in this case $r_{\text {cut }}=3 \AA$. For the crystalline phase with correlations over the complete fitting range, we used the PDF refinement result obtained for $3<r<20 \AA$ (see Table 6). Only a scale factor and the correlated motion parameter $\delta$ were allowed to refine. For the amorphous phase with correlations only out to $r_{\text {cut }}=3 \AA$, we refined all structural parameters except the atomic positions. The refinement is shown in Fig. 7 and the resulting parameters are listed in Table 3. First we observe that for the range $3<r<20 \AA$ the refinements yields the same agreement of $R_{w p}=9.6 \%$ as for the single phase refinement over the same range. Considering we only varied the scale and the parameter $\delta$, this is expected. The significant improvement is seen in the low $r$ range and overall the refinement has improved from $R_{w p}=20.6 \%$ for the single phase model to $R_{w p}=12.6 \%$ for the two phase model. From the $\mathrm{Si}-\mathrm{O}$ bond lengths listed in Table 3 we see that the two phases are structurally very similar. The scale factors result in a phase fraction of the amorphous phase of $9.3 \%$. However, closer inspection of Fig. 7 shows that despite the large improvement by adding a second phase, the low $r$ region of the experimental PDF is still not modeled as well as

Table 3. PDF results of two phase refinement. The units for the bond lengths are $\AA$. The numbers in parentheses are the estimated standard deviation on the last digit. For more details see text.

\begin{tabular}{lll}
\hline Phase & Crystalline & Amorphous \\
\hline Correlation range & $1<r<20 \AA$ & $1<r<3 \AA$ \\
$\mathrm{Si}-\mathrm{O}(1)$ & $1.60821(3)$ & $1.60809(3)$ \\
$\mathrm{Si}-\mathrm{O}(2)$ & $1.61336(5)$ & $1.61361(4)$ \\
$\mathrm{Scale}$ & $1.096(3)$ & $0.1129(6)$ \\
$R_{w p}(1-20 \AA)$ & $12.6 \%$ & \\
$R_{w p}(3-20 \AA)$ & $9.6 \%$ \\
$R_{w p}(1-3 \AA)$ & $20.1 \%$ & \\
\hline
\end{tabular}


the data at $r>3 \AA$. This is most likely due to the lack of the model to account for the more complicated correlated motion present in $\mathrm{SiO}_{4}$ tetrahedra. Several tests with different models for the correlated motion in the amorphous phase (see Eq. (4)) resulted in similar results, however, the corresponding scale factors yielded phase fractions between 5 to $10 \%$. Regardless, these two phase refinements provide clear evidence for an amorphous silica phase present in Fontainebleau sandstone. Obtaining more detailed quantitative information regarding phase fractions as well as structure requires more development of our modeling techniques, specifically related to the modeling of correlated motion. These developments are currently under way.

\section{Summary}

There are plenty of examples of the PDF technique being applied to disordered crystalline materials, glasses as well as liquids. In this paper we have addressed the question how the PDF method can be used to extract information about systems that contain crystalline as well as amorphous structural phases. In the case of the reinforced BMG we demonstrated that the crystalline phase consisting of tungsten particles and the glassy BMG phase can be separated. This opens the door to the study of the atomic structure for the two components individually and experiments are planned to use the PDF technique to look at the structural response of both phases to external loading. In the second example, we showed structural refinement results of an unexpected two phase system, Fontainebleau sandstone. This was thought to be pure crystalline quartz, but using the PDF technique clear evidence of a amorphous silica phase was found and modeled using two phase refinements. One problem, however, especially in more complex systems is to answer the question if certain features observed in the PDF indeed point to an amorphous contribution or are they in fact related to disorder in the crystalline phase? In our two examples this question was relatively easy to answer: In case of the BMG the material was know to contain a glassy and a crystalline phase. In case of the sandstone, the type of deviations from the crystalline model observed in the PDF are inconsistent with types of disorder that could be present in quartz. In general, however, complementary techniques such as electron microscopy or single crystal diffraction might be needed to really understand the local structure of the material.

Finally we like to draw the readers attention to the fact that total scattering or the PDF technique provide a method to unravel the atomic structure of materials ranging from liquids, over glasses to disordered crystalline materials as well as mixtures thereof as shown in this paper. The authors are convinced that the PDF method will become the structural tool of choice for complex materials.

Acknowledgments. We would like to thank Bernard Zinszner at the Institut Français du Pétrole for the Fontainebleau sandstone samples as well as Boonrat Lohwongwatana for his help in making the BMG samples. The BMG work was supported by the Center for Structural Amorphous Metals funded through Army Research Office grant no.
DAAD19-01-0525. This work has also benefited from the use of the Lujan Center at Los Alamos Neutron Science Center, funded by DOE Office of Basic Energy Sciences and Los Alamos National Laboratory funded by Department of Energy under contract W-7405-ENG36. The upgrade of NPDF has been funded by NSF through grant DMR 00-76488. TP, TD and JT acknowledge their support through the Los Alamos National Laboratory LDRD program.

\section{References}

Balch, D. K.; Ustundag, E.; Dunand, D. C.: Elastoplastic load transfer in bulk metallic glass composites containing ductile particles. Metall. Trans. A34(9) (2003) 17871797.

Billinge, S. J. L.; Proffen, Th.: The total scattering homepage 2004. http://www.totalscattering.org/.

Bruck, H. A.; Christman, T.; Rosakis, A. J.; Johnson, W. L.: QuasiStatic Constitutive Behavior of $\mathrm{Zr}_{41.25} \mathrm{Ti}_{13.75} \mathrm{Ni}_{10} \mathrm{Cu}_{12.5} \mathrm{Be}_{22.5}$ Bulk Amorphous-Alloys. Scripta Metall. 30 (1994) 429.

Choi-Yim, H.; Busch, R.; Köster, U.; Johnson, W. L.: Synthesis and Characterization of Particulate Reinforced $\mathrm{Zr}_{57} \mathrm{Nb}_{5} \mathrm{Al}_{10} \mathrm{Cu}_{15.4} \mathrm{Ni}_{12.6}$ Bulk Metallic Glass Composites. Acta Mater. 47 (1999) 2455-2462.

Choi-Yim, H.; Corner, R. D.; Szuecs, F.; Johnson, W. L.: Processing, microstructure and properties of ductile metal particulate reinforced $\mathrm{Zr}_{57} \mathrm{Nb}_{5} \mathrm{Al}_{10} \mathrm{Cu}_{15.4} \mathrm{Ni}_{12.6}$ bulk metallic glass composites. Acta Mater. 50 (2002) 2737-2745.

Clausen, B.; Lee, S. Y.; Ustundag, E.; Aydiner, C. C.; Conner, R. D.; Bourke, M. A. M.: Compressive yielding of tungsten fiber reinforced bulk metallic glass composites. Scripta Mater. 49(2) (2003) $123-138$.

Conner, R. D.; Dandliker, R. B.; Johnson, W. L.: Mechanical Properties of Tungsten and Steel Fiber Reinforced $\mathrm{Zr}_{41.25} \mathrm{Ti}_{13.75} \mathrm{Cu}_{12.5} \mathrm{Ni}_{10} \mathrm{Be}_{22.5}$ Metallic Glass Matrix Composites. Acta Mater. 46 (1998) 60896102.

Darling, T. W.; Tencate, J. A.; Brown, D. W.; Clausen, B.; Vogel, S. C.: Neutron diffraction study of the contribution of grain contacts to nonlinear stress-strain behavior. Geophys. Res. Lett. 31, No. 16 (2004) L16604 1-4.

Dragoi, D.; Ustundag, E.; Clausen, B.; Bourke, M. A. M.: Investigation of thermal residual stresses in tungsten-fiber/bulk metallic glass matrix composites. Scripta Mater. 45(2) (2001) 245-252.

Egami, T.; Billinge, S. J. L.: Underneath the Bragg-Peaks: Structural Analysis of Complex Materials. Elsevier Science B.V. Amsterdam 2003.

Gilbert, C. J.; O. Ritchie, R.; Johnson, W. L.: Fracture Toughness and Fatigue-Crack propagation in a ZrTiNiCuBe Bulk Metallic Glass. Appl. Phys. Lett. 71(4) (1993) 476-478.

Hays, C. C.; Kim, C. P.; Johnson, W. L.: Improved mechanical behavior of bulk metallic glasses containing in situ formed ductile phase dendrite dispersions. Mater. Sci. Eng. A304 (2001) 650-655.

Jeong, I.-K.; Heffner, R. H.; Graf, M. J.; Billinge, S. J. L.: Lattice Dynamics and Correlated Atomic Motion from the Atomic Pair Distribution Function. Phys. Rev. B67 (2003) 104301.

Jeong, I.-K.; Proffen, Th.; MohiuddinJacobs, F.; Billinge, S. J. L.: Measuring Correlated Atomic Motion using X-Ray Diffraction. J. Phys. Chem. A103 (1999) 921-924.

Larson, A. C.; Von Dreele, R. B.: General Structure Analysis System (GSAS). Report LAUR 86-748 Los Alamos National Laboratory 2000.

McGreevy, R. L.; Pusztai, L.: Reverse Monte Carlo Simulation: a New Technique for the Determination of Disordered Structures. Mol. Simul. 1 (1988) 359-367.

Nield, V. M.; Keen, D. A.: Diffuse Neutron Scattering from Crystalline Materials. Oxford University Press Oxford 2001.

Page, K. L.; Proffen, Th.; McLain, S. E.; Darling, T. W.; TenCate, J. A.: Local Atomic Structure of Fontainebleau Sandstone: Evidence for an Amorphous Phase ? Geophys. Res. Lett. 31 (2004) L24606.

Peker, A.; Johnson, W. L.: A Highly Processable Metallic-Glass $\mathrm{Zr}_{41.2} \mathrm{Ti}_{13.8} \mathrm{Cu}_{12.5} \mathrm{Ni}_{10.0} \mathrm{Be}_{22.5}$. Appl. Phys. Lett. 63(17) (1993) 2342-2344.

Peterson, P. F.; Gutmann, M.; Proffen, Th.; Billinge, S. J. L.: PDFgetN: A User-Friendly Program to Extract the Total Scattering Structure Function and the Pair Distribution Function from Neutron Powder Diffraction Data. J. Appl. Cryst. 33 (2000) 1192. 
Proffen, Th.: Analysis of Occupational and Displacive Disorder using the Atomic Pair Distribution Function: a Systematic Investigation. Z. Kristallogr. 215 (2000) 661-668.

Proffen, Th.; Billinge, S. J. L.: PDFFIT, a Program for Full Profile Structural Refinement of the Atomic Pair Distribution Function. J. Appl. Cryst. 32 (1999) 572-575.

Proffen, Th.; Billinge, S. J. L.: PDFFIT Users Guide. Manual Version 1.3 MSU 2003.

Proffen, Th.; Billinge, S. J. L.; Egami, T.; Louca, D.: Structural analysis of complex materials using the atomic pair distribution function - a practical guide. Z. Kristallogr. 218 (2003) 132-143.

Proffen, Th.; Egami, T.; Billinge, S. J. L.; Cheetham, A. K.; Louca, D.; Parise, J. B.: Building a high resolution total scattering powder diffractometer - Upgrade of NPD at MLNSC. Appl. Phys. A 74 (2002) S163-S165.

Proffen, Th.; Page, K. L.: Obtaining Structural Information from the Atomic Pair Distribution Function. Z. Kristallogr. 219 (2004) $130-135$.
Proffen, Th.; Petkov, V.; Billinge, S. J. L.; Vogt, T.: Chemical short range order obtained from the atomic pair distribution function. $\mathrm{Z}$. Kristallogr. 217 (2002) 4750.

Szuecs, F.; Kim, C. P.; Johnson, W. L.: Mechanical Properties of $\mathrm{Zr}_{56.2} \mathrm{Ti}_{13.8} \mathrm{Nb}_{5.0} \mathrm{Cu}_{6.9} \mathrm{Ni}_{5.6} \mathrm{Be}_{12.5}$ Ductile Phase Reinforced Bulk Metallic Glass Composite. Acta Mater. 49 (2001) 1507-1513.

TenCate, J. A.; Shankland, T. J.: Slow dynamics in the nonlinear elastic response of Berea sandstone. Geophys. Res. Lett. 23 (1996) 3019-3022.

Tucker, M. G.; Dove, M. T.; Keen, D. A.: Application of the Reverse Monte Carlo Method to Crystalline Materials. J. Appl. Cryst. 34 (2001) 630-638.

Waniuk, T. A.; Schroers, J.; Johnson, W. L.: Critical Cooling Rate and Thermal Stability of $\mathrm{Zr}-\mathrm{Ti}-\mathrm{Cu}-\mathrm{Ni}-\mathrm{Be}$ Alloys. Appl. Phys. Lett. 78(9) (2001) 1213-1215.

Welberry, T. R.: Diffuse Xray Scattering And Models Of Disorder. International Union of Crystallography Monographs on Crystallography. Oxford University Press Oxford 2004. 